\title{
The Inspection of Spheroidized Annealing on SCM435 Cold-Forging Quality Steel Wires with Protective Atmosphere
}

\author{
Chih-Cheng Yang1, Nan-Hua Lu \\ ${ }^{1}$ Department of Mechanical Engineering and Automation Engineering, Kao Yuan University, Taiwan \\ ${ }^{2}$ Graduate School of Fasteners Industry Technology, Kao Yuan University, Taiwan \\ Email: t30043@cc.kyu.edu.tw
}

How to cite this paper: Yang, C.-C. and $\mathrm{Lu}$, N.-H. (2019) The Inspection of Spheroidized Annealing on SCM435 Cold-Forging Quality Steel Wires with Protective Atmosphere. Materials Sciences and Applications, 10, 677-686.

https://doi.org/10.4236/msa.2019.1011048

Received: September 19, 2019

Accepted: October 27, 2019

Published: October 30, 2019

Copyright () 2019 by author(s) and Scientific Research Publishing Inc. This work is licensed under the Creative Commons Attribution International License (CC BY 4.0).

http://creativecommons.org/licenses/by/4.0/ (c) (i) Open Access

\begin{abstract}
A cold-forging quality steel rod is usually applied for manufacturing wire which is generally produced by drawing wire coil into wire, followed by spheroidized annealing treatment to achieve the necessary formability for cold forging. The subcritical and intercritical processes are usually used to spheroidize the steel wires. The cold-forging quality SCM435 alloy medium carbon steel wires are widely used to manufacture high tension bolts for mechanical and heavy industry. In this study, the spheroidized annealing experiments on SCM435 alloy steel wires are conducted in a commercial bell furnace with a protective atmosphere of nitrogen or hydrogen. The mechanical properties of annealed steel wires are measured by tensile and Rockwell hardness tests and the process capability is evaluated. It is experimentally revealed that, for SCM435 alloy medium carbon steel wires, the wire quality with intercritical annealing is much better than that with subcritical annealing and is markedly affected by furnace atmospheres. The intercritical annealing quality on SCM435 alloy steel wire in hydrogen atmosphere furnace is better than in nitrogen atmosphere furnace. A comparison between the results obtained using the intercritical annealing with hydrogen atmosphere and the measures using the subcritical annealing shows that the intercritical annealing effectively improves the performance measures of low strength and high ductility over their values at the subcritical annealing. The results presented in this study could be a reference for fasters wire manufacturers.
\end{abstract}

\section{Keywords}

Formability, Intercritical Annealing, SCM435 Alloy Steel Wire, Hydrogen Atmosphere Furnace 


\section{Introduction}

The SCM435 alloy medium carbon steel wires are widely used to fabricate high strength bolts and machine parts. The wires are usually produced by drawing, followed by spheroidized annealing to achieve the necessary formability for cold forging. The majority of all spheroidizing activity is performed to improve the ductility of steel [1] [2] [3] for cold formability. The subcritical and intercritical processes are usually used for the spheroidized annealing of steel wires [3] with a protective atmosphere of nitrogen or hydrogen [4] [5]. The subcritical annealing treatment is simply heating the steel wires to a temperature below $\mathrm{Ac}_{1}$ temperature and holding at this temperature which the annealing time may be very long. The intercritical annealing treatment consists of a duration holding at a temperature between $\mathrm{Ac}_{1}$ and $\mathrm{Ac}_{3}$ temperatures followed by slow cooling or holding at a temperature below $\mathrm{Ac}_{1}$ temperature. The spheroidized annealing usually consumes a large amount of time and energy.

To improve spheroidization of the steel wires, some studies have been conducted. O'Brien and Hosford [6] investigated the spheroidization of the medium carbon steels, AISI 1541 and AISI 4037, used in the bolt industry with intercriti$\mathrm{cal}$ and subcritical cycles and indicated that the subcritical annealing could require shorter process time than the intercritical annealing for achieving higher formability of the medium carbon steel. Introducing defects into cementites by severe plastic deformation is one of the effective methods for increasing the spheroidization speed. Ko et al. [7] proposed a method of continuous shear drawing for industrial applications to steel wire manufacturing and compared the spheroidization behavior of medium-carbon steel processed by continuous shear drawing to that processed by conventional drawing. Joo et al. [8] investigated the effect of the non-circular drawing sequence on spheroidization behavior of the medium carbon steel wire. It was indicated that the cementite in medium carbon steel spheroidizes much more easily after a severe non-circular drawing, and SEM results also revealed that the prior cold working could increase the spheroidization ratio with cold workability improved by subcritical annealing. Gul' et al. [9] developed a method for a more intense spheroidization of cementites to accelerate spheroidization. Spheroidization is induced by nonisothermal holding at high temperatures using an internal heat source. The results show that the spheroidized annealing temperature and prolonged heating time have the greatest effect on the mechanical properties of steel wires. Ji et al. [10] investigated the effect of subcritical annealing temperature on microstructure and mechanical properties of SCM435 steel through changing the heating and soaking temperature. Lv et al. [11] investigated the spheroidizing behavior of cementite in $\mathrm{Fe}-0.8 \mathrm{C}$ mass\% steel during cyclic heat treatment and indicated that cyclic heat treatment method could effectively accelerate spheroidization of cementite.

For low carbon steel wires, Yang and Liu [12] conducted the experiment by using Taguchi method to obtain optimum subcritical annealing conditions to improve the mechanical properties of AISI 1022 steel wire for cold forming. Yang 
and Wang [13] studied experimentally on AISI 10B21 steel wires also by using Taguchi method to obtain optimal subcritical annealing conditions to improve the mechanical properties of steel wires for cold forming. Their results show that the spheroidized annealing temperature and prolonged heating time have the greatest effects on the mechanical properties of steel wires. Okonogi and Yamazaki [14] investigated the effects of microstructure on mechanical properties of spheroidized annealing medium carbon steel wire rods and control methods of microstructure of JIS SWRCH40K medium carbon steel wire rods. The fine dispersed cementites and coarse ferrite grain were observed in the medium carbon steel wire rods manufactured by isothermal transformation treatment.

Annealing can be performed in batch or continuous furnaces. The most common type of furnace used for annealing of steel coils is a bell type furnace [5]. In this study, the intercritical annealing experiment (at an annealing temperature of $770^{\circ} \mathrm{C}$ ) is conducted in a commercial bell furnace to investigate spheroidizing behavior of SCM435 alloy steel wires in comparison with those at subcritical annealing temperature of $680^{\circ} \mathrm{C}$ and $720^{\circ} \mathrm{C}$ ). The furnace atmosphere also becomes important to effective heat treating. For hydrogen atmosphere, its thermal conductivity is approximately seven times that of nitrogen. To investigate the effect of protective atmosphere, the wires are spheroidized with atmospheres of nitrogen and hydrogen, respectively. Mechanical properties are measured by tensile and Rockwell hardness tests and the process capability is evaluated as well.

\section{Spheroidized Annealing Experiments}

In this study, the SCM435 alloy steel wire is spheroidizing annealed after colddrawn at $13.1 \%$ deformation. The steel wire coil ( $\varnothing 9.0 \mathrm{~mm}$, Al-killed) is manufactured by China Steel Corporation, Kaohsiung, Taiwan. Its chemical composition is shown in Table 1.

For the intercritical process, the wires are heated into the intercritical temperature of $770^{\circ} \mathrm{C}$, which is between the $\mathrm{Ac}_{1}$ and $\mathrm{Ac}_{3}$ temperatures as illustrated in Figure 1, for 2.5 hours and followed by slow cooling to the temperature of $665^{\circ} \mathrm{C}$ which is below $\mathrm{Ac}_{1}$ temperature. It takes 21 hours in total. The experimental tests on the alloy steel wires are carried out in an A210 bell-type furnace (Rad-Con Inc., Cleveland, OH, USA) with protective atmospheres of nitrogen and hydrogen, respectively.

With protective atmospheres of hydrogen in the same commercial bell furnace, the subcritical annealing treatment was conducted by heating the wires to the temperature as $680^{\circ} \mathrm{C}$ and $720^{\circ} \mathrm{C}$, which are below $\mathrm{Ac}_{1}$ temperature as shown in Figure 1, for 5 hours and followed by slow cooling to the temperature of $450^{\circ} \mathrm{C}$. The cycle time is about 22 hours.

In this study, the mechanical properties of the spheroidized annealed wires, such as tensile strength, ductility and hardness, are investigated. The tensile test is used as a measure of ductility by calculating the elongation of the specimen 
Table 1. Chemical composition of SCM435 alloy steel wires (wt\%).

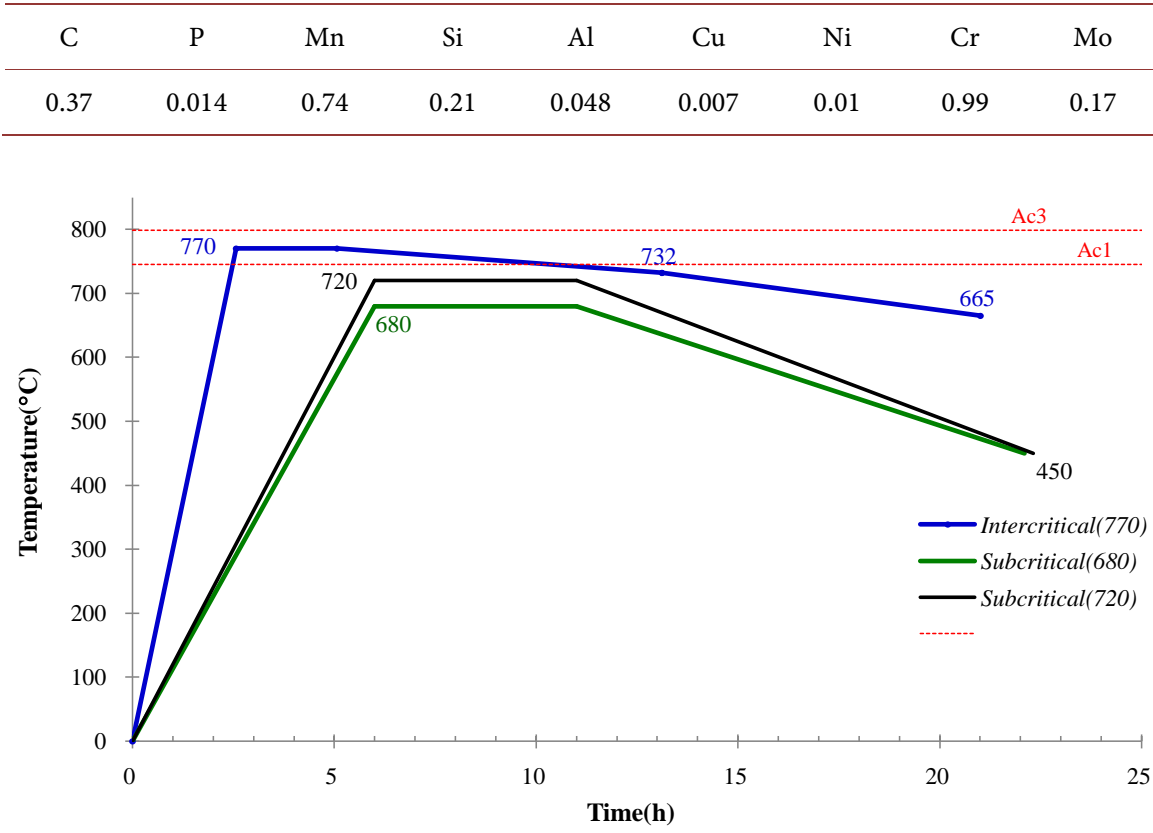

Figure 1. Spheroidized annealing procedures.

upon fracture [15]. The tensile tests are conducted on a 20 ton universal testing machine under a constant ram speed of $7 \mathrm{~mm} / \mathrm{min}$ at room temperature. The dimensions of the tensile specimen are $\varnothing 8.39 \mathrm{~mm} \times \mathrm{L} 200 \mathrm{~mm}$. The Rockwell hardness values are measured at 6 points on each specimen. Each test trial, including ten specimens which are taken from distinct regions in the furnace, is followed by a fabrication process.

Process capability is a measure of the nature behavior of the process after special causes of variation are eliminated, and the basic purpose is to compare the "normal variation" of the process against the design tolerances to assess whether the process can meet those specifications [15]. Spheroidized annealing primarily provides a needed ductility for cold forging which the ductility of the steel wire is increased and its strength and hardness is reduced too. As the company assigned, the ductility of the annealed wires should be exceeding 0.30 . For the ductility of the annealed wires, since only lower specification limit is necessary, the process-capability ratio [15]

$$
C P L=\frac{\mu-L S L}{3 S},
$$

is measured, where $\mu$ is the process average, $L S L$ is the lower specification limit and $S$ is the process standard deviation of each trial.

Through spheroidized annealing, the ductility of the steel wire may be increased, and its strength is simultaneously reduced. However, the annealed steel wire has to supply a certain strength for cold heading. The tensile strength of the annealed wires should not exceeding $620 \mathrm{MPa}$ and the hardness not exceeding $90 \mathrm{HRB}$, which are assigned by the company. Since only upper specification lim- 
it is necessary for the tensile strength and hardness of the annealed wires, the process-capability ratio is measured as [15]

$$
C P U=\frac{U S L-\mu}{3 S},
$$

where $U S L$ is the upper specification limit of each trial.

\section{Results and Discussion}

Figure 2(a) and Figure 2(b) show the microstructures of undrawn and drawn steel wires, respectively. They are not yet spheroidized annealed. For a non-treated drawn wire, the tensile strength of $1005.0 \mathrm{MPa}$, the ductility of 0.109 and the hardness of 105.0 HRB are measured. The high strength, hardness and poor ductility are mainly due to heavy plastic work of drawing. Spheroidization is a process to produce a microstructure in which contains sphere-like cementitie particles, as shown in Figure 2(c). The globular structure obtained increases formability of the steel wire.

When the wires are manufactured following the spheroidized annealing procedures displayed in Figure 1, the experimental results of the tensile strength, ductility and hardness (mean, $\mu$; standard deviation, $S$; and process-capability ratio, CPU or CPL) of the spheroidized annealed steel wires are shown in Tables $2-4$, respectively. It is revealed that, in comparison to non-treated wires, the mean tensile strength is reduced more than $300 \mathrm{MPa}$, the mean hardness is also decreased at least $10 \mathrm{HRB}$, and the mean ductility is improved over 0.12 for the annealed wires. However, the effectiveness of the four annealing processes is obviously different. In addition, for a commercial bell furnace, the charge weight of wires may be up to 30 tons and the obvious variation of annealing quality may be due to the convection flow in the furnace.

Variation is the inability to perform a task consistently according to a specification [15]. Observe Figure 3 and Figure 4, where the natural variations of the process [15] of the four tests together with the non-treated experiment, including the given specification limit/spans, are plotted in the figures respectively for

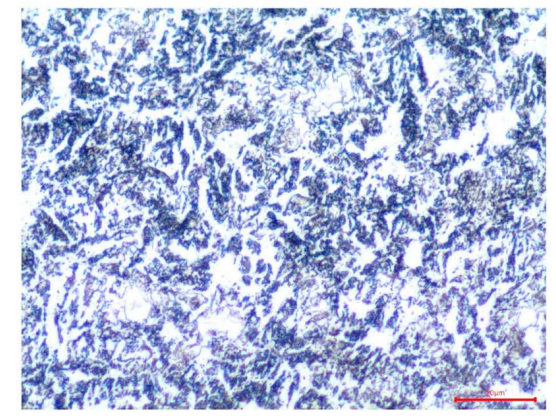

(a)

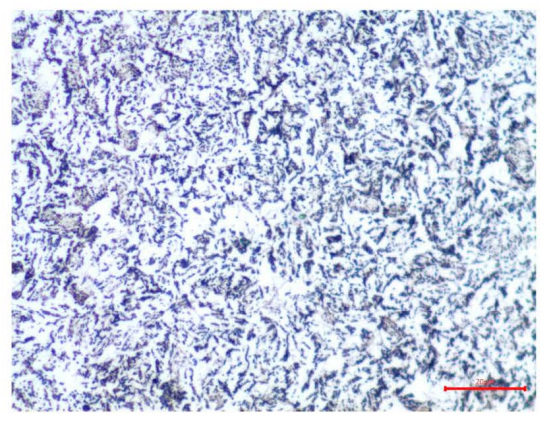

(b)

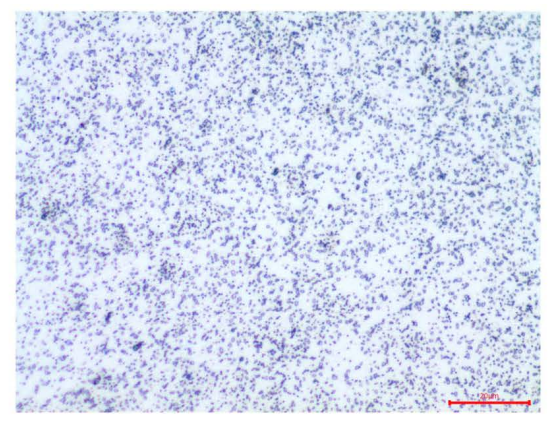

(c)

Figure 2. Microstructures of SCM435 alloy steel wire $(\times 500)$. (a) Undrawn (Non-treated); (b) Drawn (Non-treated); (c) Intercritical annealed. 
Table 2. Experimental results for tensile strength (MPa).

\begin{tabular}{|c|c|c|c|c|c|c|c|c|c|c|c|c|c|}
\hline Exp. & $\mathrm{T} 1$ & $\mathrm{~T} 2$ & T3 & $\mathrm{T} 4$ & T5 & T6 & T7 & T8 & T9 & $\mathrm{T} 10$ & $\mu$ & $S$ & $\mathrm{CPU}$ \\
\hline Intercritical $\left(\mathrm{H}_{2}\right)$ & 512.6 & 557.4 & 608.4 & 560.3 & 555.7 & 527.5 & 507.6 & 598.9 & 600.4 & 587.4 & 561.6 & 36.95 & 0.53 \\
\hline Intercritical $\left(\mathrm{N}_{2}\right)$ & 538.9 & 586.2 & 501.3 & 597.3 & 588.5 & 605.8 & 606.6 & 621.3 & 574.0 & 586.7 & 580.6 & 35.67 & 0.37 \\
\hline Subcritical (720) & 593.5 & 628.0 & 645.7 & 624.2 & 641.4 & 628.6 & 626.6 & 644.3 & 617.1 & 631.5 & 628.1 & 15.24 & - \\
\hline Subcritical (680) & 701.5 & 679.0 & 674.5 & 705.9 & 695.4 & 691.5 & 662.9 & 656.2 & 681.2 & - & 683.1 & 16.98 & - \\
\hline Non-treated & 1014.5 & 1133.9 & 970.3 & 986.2 & 982.7 & 991.2 & 977.5 & 993.4 & 1016.0 & 984.6 & 1005.0 & 47.57 & \\
\hline
\end{tabular}

Table 3. Experimental results for ductility $\left(\varepsilon_{f}\right)$.

\begin{tabular}{|c|c|c|c|c|c|c|c|c|c|c|c|c|c|}
\hline Exp. & $\mathrm{T} 1$ & $\mathrm{~T} 2$ & $\mathrm{~T} 3$ & $\mathrm{~T} 4$ & $\mathrm{~T} 5$ & T6 & $\mathrm{T} 7$ & $\mathrm{~T} 8$ & T9 & $\mathrm{T} 10$ & $\mu$ & $S$ & CPL \\
\hline Intercritical $\left(\mathrm{H}_{2}\right)$ & 0.388 & 0.320 & 0.299 & 0.336 & 0.328 & 0.407 & 0.402 & 0.314 & 0.300 & 0.302 & 0.339 & 0.043 & 0.31 \\
\hline Intercritical $\left(\mathrm{N}_{2}\right)$ & 0.304 & 0.255 & 0.354 & 0.279 & 0.269 & 0.274 & 0.283 & 0.277 & 0.242 & 0.294 & 0.283 & 0.031 & - \\
\hline Subcritical (720) & 0.301 & 0.280 & 0.291 & 0.289 & 0.252 & 0.289 & 0.280 & 0.278 & 0.288 & 0.282 & 0.283 & 0.013 & - \\
\hline Subcritical (680) & 0.215 & 0.230 & 0.254 & 0.233 & 0.240 & 0.265 & 0.230 & 0.237 & 0.235 & - & 0.238 & 0.015 & - \\
\hline Non-treated & 0.110 & 0.095 & 0.113 & 0.117 & 0.107 & 0.106 & 0.115 & 0.107 & 0.104 & 0.112 & 0.109 & 0.007 & \\
\hline
\end{tabular}

Table 4. Experimental results for hardness (HRB).

\begin{tabular}{|c|c|c|c|c|c|c|c|c|c|c|c|c|c|}
\hline Exp. & $\mathrm{T} 1$ & $\mathrm{~T} 2$ & $\mathrm{~T} 3$ & $\mathrm{~T} 4$ & T5 & T6 & $\mathrm{T} 7$ & $\mathrm{~T} 8$ & T9 & $\mathrm{T} 10$ & $\mu$ & $S$ & $\mathrm{CPU}$ \\
\hline Intercritical $\left(\mathrm{H}_{2}\right)$ & 90.8 & 89.0 & 87.8 & 83.2 & 82.1 & 87.4 & 82.2 & 90.8 & 90.8 & 91.6 & 87.6 & 3.76 & 0.22 \\
\hline Subcritical (720) & 86.3 & 87.8 & 88.2 & 85.0 & 87.6 & 88.0 & 88.6 & 88.4 & 86.8 & 89.0 & 87.6 & 1.21 & 0.67 \\
\hline Subcritical (680) & 89.9 & 92.6 & 91.5 & 92.0 & 90.8 & 92.5 & 92.0 & 90.8 & 93.0 & 92.9 & 91.8 & 1.04 & - \\
\hline Non-treated & 106.2 & 104.0 & 106.1 & 104.5 & 106.3 & 106.3 & 105.6 & 105.4 & 101.3 & 104.6 & 105.0 & 1.55 & \\
\hline
\end{tabular}

the tensile strength together with ductility and hardness of SCM435 alloy steel wires. The properties of spheroidized annealing wires are obviously varied with various annealing processes.

\subsection{Tensile Strength}

In comparison with both subcritical processes annealing at $680^{\circ} \mathrm{C}$ and $720^{\circ} \mathrm{C}$, as shown in Table 2 and Figure 3, the mean tensile strengths of annealed wires are smaller for the two intercritical processes annealing at $770^{\circ} \mathrm{C}$, particularly with protective atmosphere of hydrogen. However, the standard deviations are larger for the two intercritical processes may be due to faster preheating rate and shorter prolonged heating time of only 2.5 hours, as shown in Figure 1.

For the four annealing processes, the mean tensile strength of annealed wires varies from 561.6 to $683.1 \mathrm{MPa}$, and the mean values of both subcritical processes are exceeding $620 \mathrm{MPa}$, as shown in Table 2 and Figure 3, particularly for the subcritical process annealing at $680^{\circ} \mathrm{C}$ which results are all exceeding the upper specification limit, as illustrated in Figure 3, due to lower annealing temperature. 


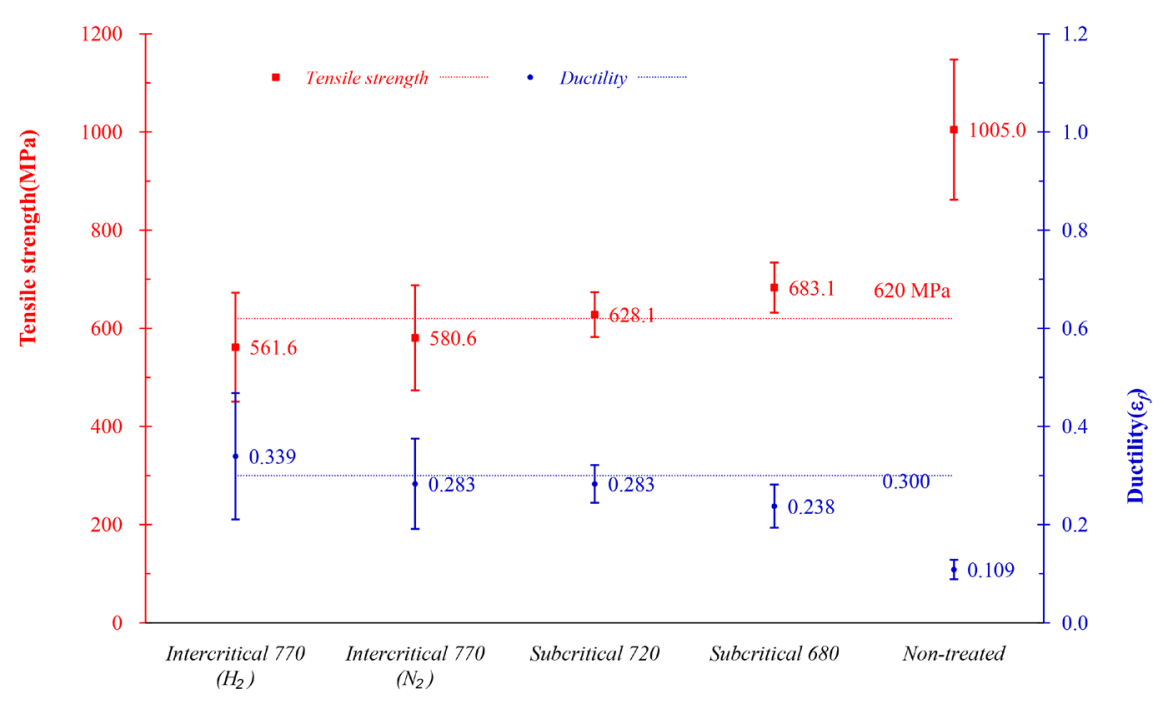

Figure 3. Natural variation of the process for tensile strength and ductility.

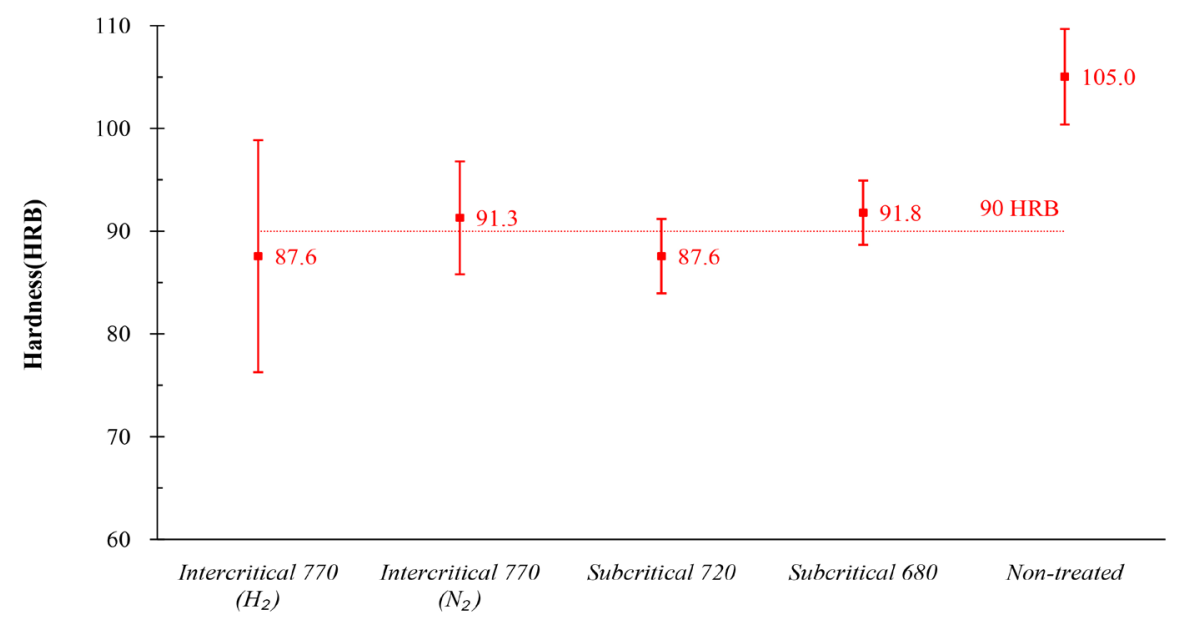

Figure 4. Natural variation of the process for hardness.

The process-capability ratios (CPU) for the four annealing processes are smaller than 1, as shown in Table 2, which mean that their natural variations of the process are larger than the tolerance band given, thus, their processes are not able to meet the specifications and incapable of ensuring $99.73 \%$ of good annealing wires [15]. However, for the intercritical process with hydrogen atmosphere, the process-capability ratio (CPU) of 0.53 , as shown in Table 2 , is the greatest. That is, for the property of tensile strength, its process capability is the best of the four annealing processes.

\subsection{Ductility}

As shown in Table 3 and Figure 3, the means of ductility vary from 0.238 to 0.339 for the four annealing processes. It is obviously that the mean ductility of wires annealing used the intercritical process with hydrogen atmosphere is the best, even better than the process with nitrogen atmosphere. However, the standard deviations for the two intercritical processes are large due to faster pre- 
heating rate and shorter prolonged heating time.

The mean values of ductility for both subcritical processes and intercritical process with nitrogen atmosphere are not exceeding 0.30, as shown in Table 3 and Figure 3, particularly for the subcritical process annealing at $680^{\circ} \mathrm{C}$ which results are not exceeding the lower specification limit, as illustrated in Figure 3, due to lower annealing temperature. The process-capability ratios (CPL) for the four annealing processes are also smaller than 1, as shown in Table 3. That is, their natural variations of the process are larger than the tolerance band given. However, for the intercritical process with hydrogen atmosphere, the processcapability ratio (CPL) of 0.31 , as shown in Table 3 , is also the greatest. Therefore, for the property of ductility, its process capability is the best of the four annealing processes.

\subsection{Hardness}

The mean values of hardness, as illustrated in Table 4, are similar for the four annealing processes. The mean hardness of annealed wires is the same for intercritical process with hydrogen atmosphere and subcritical process annealing at $720^{\circ} \mathrm{C}$, but with different standard deviation, as illustrated in Table 4 and Figure 4. The standard deviations for the intercritical processes are also large. For the intercritical process with nitrogen atmosphere and subcritical process annealing at $680^{\circ} \mathrm{C}$, their mean values of hardness are exceeding $90 \mathrm{HRB}$, as shown in $\mathrm{Ta}$ ble 4 and Figure 4

The process-capability ratios (CPU) for the four annealing processes are also smaller than 1, as shown in Table 4, thus, their natural variations of the process are larger than the tolerance band given. However, for the subcritical process annealing at $720^{\circ} \mathrm{C}$, the process-capability ratio (CPU) of 0.67 , as shown in Table 4 , is the greatest in consequence of smaller standard deviation.

In the commercial application, the bell-type furnace is usually used for the heat treatment process of spheroidized annealing medium-carbon alloy steels for cold-heading quality. The character of the bell furnace concept leads to efficient use of the furnace chamber space and the furnace can be configured for a single-stack of coils, or for multiple-stacks under one inner cover [5]. Therefore, to obtain stable annealing quality of SCM435 alloy steel wires, highly efficient convection flow in the furnace chamber space is essential.

Commercial spheroidizated annealing usually consumes a large amount of time and energy. The atmospheres used in the annealing furnace is not only to protect the steel wires being processed from chemical reactions that could occur on their surfaces (e.g. oxidation or decarburization), but also to conduct heat to the wires. The thermal conductivity of hydrogen is approximately seven times that of nitrogen. The hydrogen gas is only $1 / 14$ th the mass of nitrogen, leading to high efficiency convection in the furnace. The higher convection flow along with the properties of hydrogen itself, substantially increases the rate at which heat is delivered to the steel wires [5]. In comparison with $\mathrm{N}_{2}$, the higher heat conductivity of $\mathrm{H}_{2}$ provides an overall improvement in heat transfer and a cor- 
responding increase in annealing and cooling output. Therefore, the annealing quality of SCM435 alloy steel wires with hydrogen annealing is better than that with nitrogen annealing, as illustrated in Figure 3 and Figure 4. However, in this study, for the intercritical process, the apparently uneven quality of annealed wires may result from shorter prolonged heating time.

With increasing the subcritical annealing temperature from $680^{\circ} \mathrm{C}$ to $720^{\circ} \mathrm{C}$, as shown in Figure 3, the performance of low strength and high ductility substantially improves the formability of SCM435 alloy steel wires. In the meantime, the quality of annealed wires is more uniform due to longer prolonged heating time. However, the performance is obviously not as good as that for intercritical process annealing at temperature of $770^{\circ} \mathrm{C}$, especially for annealing with hydrogen atmosphere. Consequently, to improve the formability of SCM435 alloy steel wires with the performance of low strength and high ductility, it is effective to apply the intercritical annealing process with hydrogen atmosphere along with longer prolonged heating time to obtain more even quality of annealed wires.

\section{Conclusion}

The cold forming quality SCM435 alloy steel wires are usually used to manufacture high tension bolts for mechanical and heavy industry. The wires are usually produced by drawing, followed by spheroidized annealing to achieve the necessary formability for cold forging. Commercial spheroidizated annealing usually consumes a large amount of time and energy. In this study, the subcritical and intercritical annealing experiments on SCM435 alloy steel wires are carried out in a commercial bell furnace with a protective atmosphere of nitrogen or hydrogen. It is experimentally revealed that, for SCM435 alloy medium carbon steel wires, the wire quality with intercritical annealing is much better than subcritical annealing and is markedly affected by furnace atmospheres. The higher heat conductivity of $\mathrm{H}_{2}$ provides an overall improvement in heat transfer. Therefore, to enhance the formability of SCM435 alloy steel wires with the performance of low strength and high ductility, it is effective to apply the intercritical annealing process with hydrogen atmosphere, and leads to obtaining the mean tensile strength of $561.6 \mathrm{MPa}$, mean ductility of 0.339 and mean hardness of 87.6 HRB. However, the process-capability ratios are smaller than 1; that is, their natural variations of the process are larger than the tolerance band given. The results presented in this study could be a reference for fasters wire manufacturers.

\section{Acknowledgements}

The authors would like to acknowledge the support of Fang Sheng Screw Co., Ltd., Kaohsiung, Taiwan, for providing the materials and apparatus to carry out the spheroidized annealing experimental work.

\section{Conflicts of Interest}

The authors declare no conflicts of interest regarding the publication of this paper. 


\section{References}

[1] Hill, R. (1984) Annealing: The First Step in Cold Forming. Wire Journal International, 17, 95-97.

[2] Dhers, J., Thivard, B. and Genta, A. (1992) Improvement of Steel Wire for Cold Heading. Wire Journal International, 25, 73-76.

[3] Bramfitt, B.L. and Hingwe, A.K. (1991) Annealing of Steel. In: Heat Treating, ASM International, Materials Park, 42-55.

[4] Johnson, P. (1991) Furnace Atmospheres. In: Heat Treating, ASM International, Materials Park, 542-567.

[5] Messina, C.J. (2011) Raw Material Processing: Advances in Spheroidize Annealing of Wire Rod Using 100\% Hydrogen. Fastener Technology International, February, 26-27.

[6] O’Brien, J.M. and Hosford, W.F. (2002) Spheroidization Cycles for Medium Carbon Steels. Metallurgical and Materials Transactions A, 33, 1255-1261.

https://doi.org/10.1007/s11661-002-0226-y

[7] Ko, Y.G., Namgung, S., Shin, D.H., Son, I.H., Rhee, K.H. and Lee, D.-L. (2010) Spheroidization of Medium Carbon Steel Fabricated by Continuous Shear Drawing. Journal of Materials Science, 45, 4866-4870. https://doi.org/10.1007/s10853-010-4587-0

[8] Joo, H.S., Hwang, S.K., Baek, H.M., Im, Y.-T., Son, I.-H. and Bae, C.M. (2015) The Effect of a Non-Circular Drawing Sequence on Spheroidization of Medium Carbon Steel Wires. Journal of Materials Processing Technology, 216, 348-356.

https://doi.org/10.1016/j.jmatprotec.2014.10.001

[9] Gul, Y.P., Sobolenko, M.A. and Ivchenko, A.V. (2012) Improvement in the Spheroidizing Annealing of Low-Carbon Steel for Cold Upsetting. Steel in Translation, 42, 531-535. https://doi.org/10.3103/S0967091212060058

[10] Ji, C., Wang, L. and Zhu, M.-Y. (2015) Effect of Subcritical Annealing Temperature on Microstructure and Mechanical Properties of SCM435 Steel. Journal of Iron and Steel Research, International, 22, 1031-1036.

https://doi.org/10.1016/S1006-706X(15)30108-4

[11] Lv, Z.Q., Wang, B., Wang, Z.H., Sun, S.H. and Fu, W.T. (2013) Effect of Cyclic Heat Treatments on Spheroidizing Behavior of Cementite in High Carbon Steel. Materials Science and Engineering: A, 574, 143-148. https://doi.org/10.1016/j.msea.2013.02.059

[12] Yang, C.-C. and Liu, C.-L. (2016) Improvement of the Mechanical Properties of 1022 Carbon Steel Coil by Using the Taguchi Method to Optimize Spheroidized Annealing Conditions. Materials, 9, 693. https://doi.org/10.3390/ma9080693

[13] Yang, C.-C. and Wang, S.-T. (2018) Improvement of the Mechanical Properties of Spheroidized 10B21 Steel Coil Using Taguchi Method of Robust Design. Sensors and Materials, 30, 503-514. https://doi.org/10.18494/SAM.2018.1755

[14] Okonogi, M. and Yamazaki, K. (2017) Development of Medium Carbon Steel Wire Rods for Cold Heading by Isothermal Transformation Treatment. Nippon Steel \& Sumitomo Metal Technical Report, 116, 65-70.

[15] Ostwald, P.F. and Munoz, J. (1997) Manufacturing Processes and Systems. John Wiley \& Sons, Inc., New York. 